\title{
Effect of diacylglycerol interfacial crystallization on the physical stability of water-in-oil emulsions
}

\begin{abstract}
The influence of diacylglycerol (DAG) combined with polyglycerol polyricinoleate (PGPR) on the stability of water-in-oil (W/O) emulsions containing hydrogenated palm oil (HPO) was studied. Polarized light microscope revealed that DAG promoted HPO to crystallize at the water-oil interface, providing the combination of Pickering and network stabilization effects. It was proposed that the molecular compatibility of fatty acids in DAG with HPO accounted for the promotional effect. The interfacial crystallization of DAG together with the surface activity of PGPR led to the formation of emulsions with uniform small droplets and high freeze-thaw stability. Further exploration of physical properties indicated that the combination of DAG and PGPR dramatically improved the emulsion's viscoelasticity and obtained a larger deformation yield. Water droplets in DAG-based emulsions acted as active fillers to improve the network rigidity. Therefore, DAG is a promising material to be used as emulsifier to enhance the physical stability of $\mathrm{W} / \mathrm{O}$ emulsions.
\end{abstract}

Keyword: W/O emulsion; Diacylglycerol; Interfacial crystallization; Freeze-thaw stability 